\title{
Détermination du bilan glaciaire en zone d'accumulation par mesure in situ de la radioactivité due à Tchernobyl
}

\author{
J.-F. Pinglot et M. Pourchet *
}

Les récentes retombées dues à Tchernobyl au printemps 1986 ont déjà été identifiées au sein des glaciers de l'hémisphère Nord, où elles constituent un nouvel horizon-repère. Nous avons développé un système de spectrométrie gamma in situ pour détecter directement sur le terrain la profondeur de l'horizon Tchernobyl.

Cet horizon est utilisé, en zone d'accumulation, pour déterminer les bilans glaciaires.

Au col du Dôme du Goûter (Massif du Mont Blanc, $4250 \mathrm{~m}$ ), la couche radioactive due à Tchernobyl atteint 100 fois la radioactivité naturelle, soit $23 \mathrm{~Bq} \cdot \mathrm{kg}^{-1}$, valeur comparable aux niveaux-repères dus aux retombées des essais thermonucléaires atmosphériques (1954 et 1962-63) (fig. 1) [1].

Le détecteur à scintillation (NaITI de $76 \times 76 \mathrm{~mm}$ ) parcourt les trous de carottage par paliers successifs de cinq minutes (temps total d'exploration: $1 \mathrm{~h}$ ) (fig. 2) [2], [3].

Le spectromètre gamma associé au détecteur possède une sélection d'amplitude centrée sur le photo-pic à $662 \mathrm{Kev}$ du Césium-137 (période 29,5 ans).

La détection de la couche radioactive a été réalisée dans 5 trous de carottage, sur le glacier du Mont de Lans (Massif des Ecrins : $3417 \mathrm{~m}$ ). La figure 3 présente les résultats concernant l'un des carottages - T3 - effectué le 25 octobre 1988. Le taux de comptage in situ du détecteur indique un maximum de radioactivité, centré à 2 mètres, qui correspond à Tchernobyl. Les mesures des radioactivités béta-globale et gamma sur échantillons recoupent parfaitement les mesures gamma in situ (voir les figures à la page suivante).
Le bilan glaciaire a été déterminé en plusieurs sites, entre le $1^{\text {er }}$ mai 1986 et le 25 octobre 1988 . Pour le site T3, le bilan s'élève à $0,76 \mathrm{~m}$ d'eau ( $1,65 \mathrm{~m}$ de neige). En ce même site, l'évolution du manteau neigeux a été examinée par le détecteur in situ à plusieurs dates. L'accumulation a été de $0,52 \mathrm{~m}$ d'eau entre le 10 mars et le 14 juin 1988 , suivi d'une ablation de $1,05 \mathrm{~m}$ d'eau entre le 14 juin et le 24 octobre.

Une relation linéaire entre le taux de comptage in situ et la radioactivité spécifique de la neige a été établie et permet de déterminer la sensibilité du détecteur $\left(1,43 \mathrm{~Bq} / \mathrm{Bq} \cdot \mathrm{kg}^{-1}\right)$, ainsi que le bilan de retombées en Césium-137 dû à Tchernobyl.

Trois stations ont des bilans sensiblement égaux, de l'ordre de $400 \mathrm{~Bq} \cdot \mathrm{m}^{-2}$, bilan déjà mesuré au Lac Lérié très proche $\left(420 \mathrm{~Bq} \cdot \mathrm{m}^{-2}\right)$ [4] ainsi qu'au Col du Dôme (massif du Mont Blanc) (538 $\mathrm{Bq} \cdot \mathrm{m}^{-2}$ ) [1]. Ces bilans de retombées présentent une valeur intermédiaire entre les bilans moyens de $5000 \mathrm{~Bq} \cdot \mathrm{m}^{-2}$ mesurés sur des glaciers suisses [5] et de $8 \mathrm{~Bq} \cdot \mathrm{m}^{-2}$ au Gröenland [6] et [7].

(*) Laboratoire de glaciologie et de géophysique de l'environnement du CNRS, BP 96, 38402 Saint-Martin d'Hères. Cedex (France) Tél. 76425872 - Télex 980131 LGGE

\section{Glacial mass balance determination in the accumulation zone by in situ measurements of Tchernobyl radioactivity}

Glacial mass balance determination in the accumulation zone is conducted by the determination of the water equivalent of the snow cover. Radioactive fallout due to the Tchernobyl accident on 26 april 1986 have already been identified in Northern hemisphere glaciers where it provides a new reference-level. We developped an in situ gamma-ray spectrometer in order to detect - directly in the field - the depth of the Tchernobyl layer. 


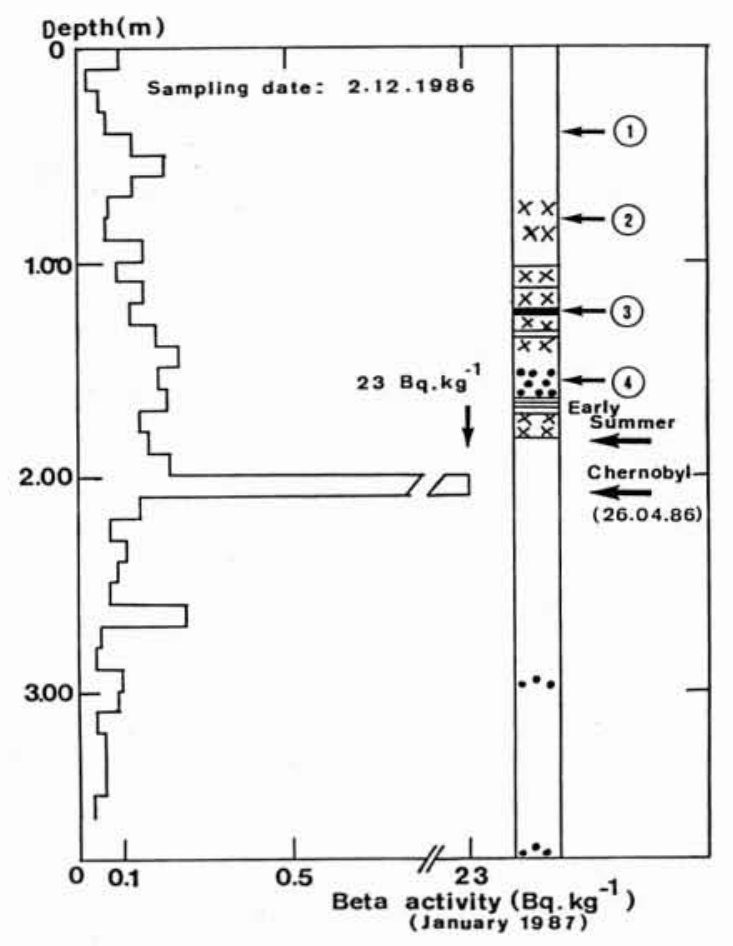

1. Radioactivité béta-globale au col du Dôme en fonction de la profondeur
(1) névé,
(2) névé transformé,
(3) glace,
(4) lentilles de glace.

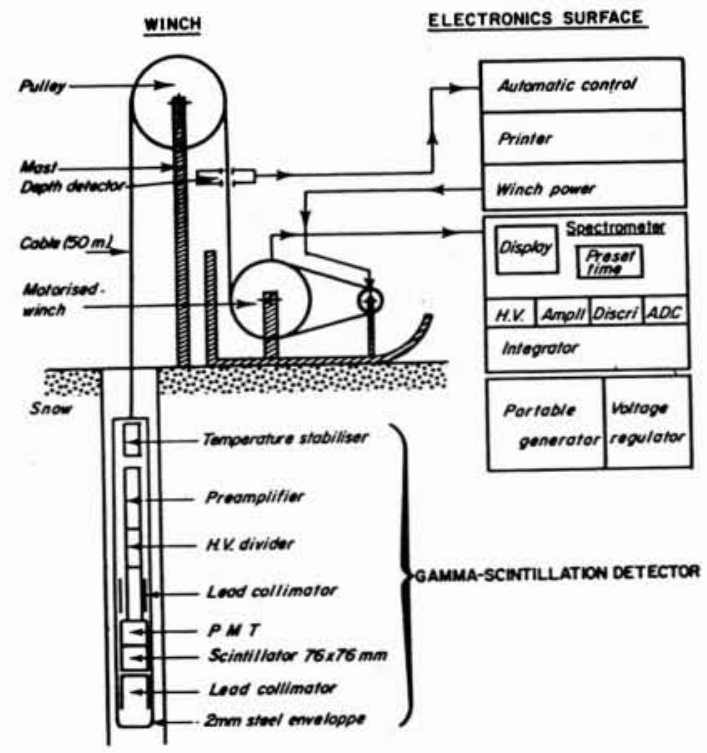

2. Spectromètre gamma in situ pour trou de carottage dans les glaciers.

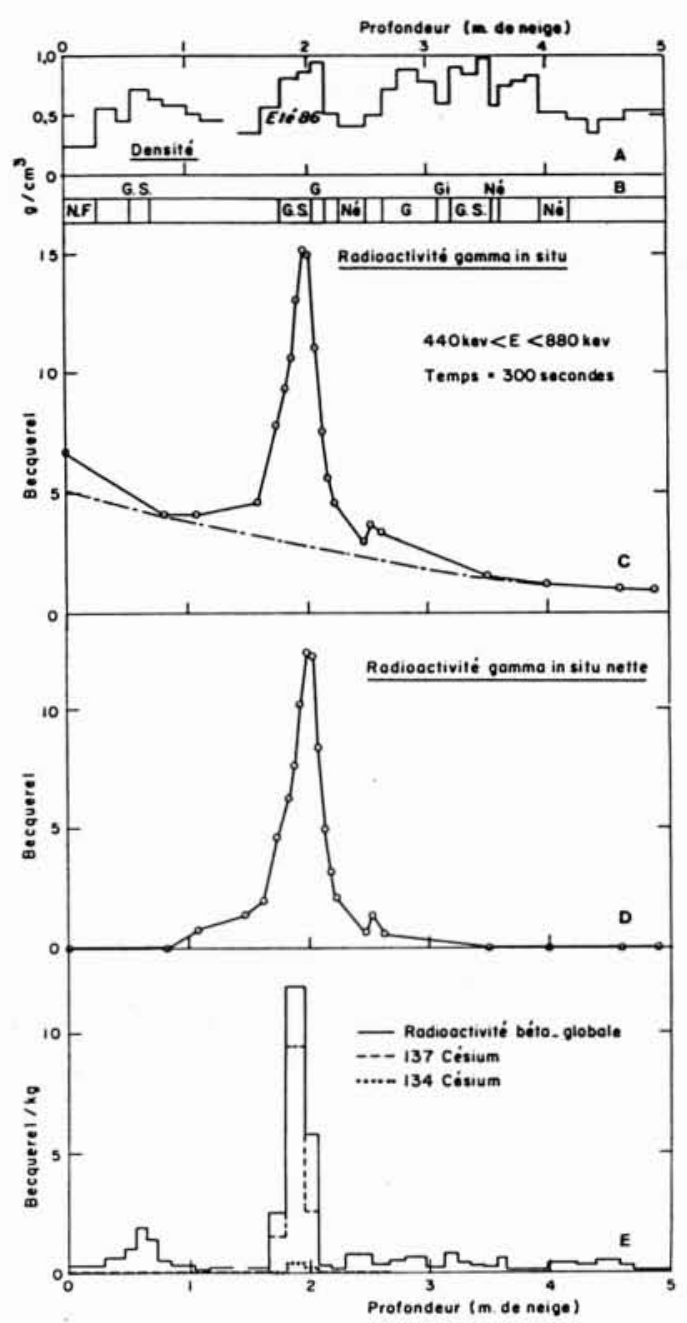

3. Mesures de densité (A), stratigraphie (B), radioactivité gamma in situ (C) et (D), radioactivité béta-globale, Césium-137, Césium-134 (E) en fonction de la profondeur en neige.

Légende de la stratigraphie (B): N.F.: Neige fraîche; G : glace; $\mathrm{S}$ : sale ; Né : névé ; Gi : givre; espaces vides : névé très transformé.

\section{Conclusion}

Le repérage direct en profondeur de la couche de neige radioactive due à Tchernobyl s'avère être une méthode sûre de datation absolue dans la zone d'accumulation des glaciers.

De plus, ces mesures de spectrométrie gamma in situ ont permis la détermination des bilans glaciaires et des retombées en Césium-137, ainsi que leurs variations spatiotemporelles. 


\section{Références}

[1] M. Pourchet, J.-F. Pinglot, L. Reynaud et G. HOLDSWORTH. - Journal of Glaciology, 34, $\mathrm{n}^{\circ} 117,1988$, p. 183-187.

[2] J.-F. Pinglot et M. Pourchet. - I.A.E.A. Publ. $n^{o}$ SM252/55, 1981, p. 161-172.

[3] J.-F. Pinglot et M. Pourchet, - C.R. Acad. Sci., t. 309, Série II, 1989 , p. 365-370.

[4] M. A. Melières, M. Pourchet, J.-F. Pinglot, R. Bouchez et M. PiBoulE. - Journal of Geophysical Research, 93, $\mathrm{n}^{\circ}$ D6, 1988, p. 7055-7061.

[5] W. Haeberli, H. Gaggeler, U. Baltensperger, D. Jost et U. SCHOTTERER. - Annals of Glaciology, $\mathrm{n}^{\circ} 10,1988, \mathrm{p} .48$ 51

[6] C. I. Davidson, J.-R. Harrington, M. J. Stephenson, M. C. Monagham, J. Pudykitewcz et W. R. Schell. - Science, $237, \mathrm{n}^{\circ} 4815,1987$, p. $633-634$.

[7] J. Dıвв. - Geophysical Research Letters, 16, $\mathrm{n}^{\circ} 9,1989$, p. $987-990$. 


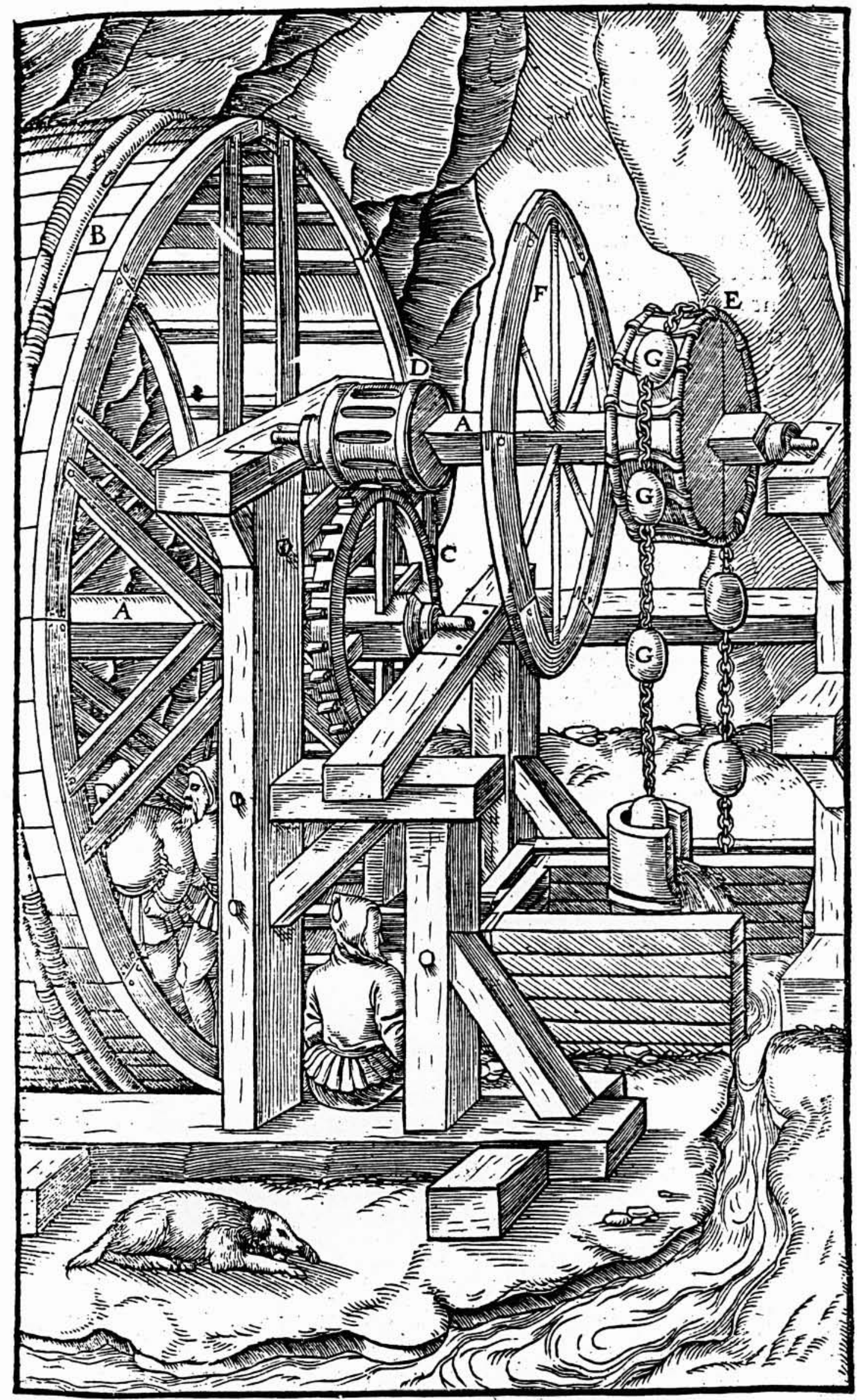

Extrait de: Agricola - De re metallica (1556)

LA HOUILLE BLANCHE/N 5-1990 